\title{
Aberrant Salience Is Related to Reduced Reinforcement Learning Signals and Elevated Dopamine Synthesis Capacity in Healthy Adults
}

\author{
Rebecca Boehme, ${ }^{1}$-Lorenz Deserno, ${ }^{1,2,3}$-Tobias Gleich, ${ }^{1}$ Teresa Katthagen, ${ }^{1}$ Anne Pankow, ${ }^{1}$ Joachim Behr $, 1,4,5$ \\ Ralph Buchert, ${ }^{1}$ D Jonathan P. Roiser, ${ }^{6}$ Andreas Heinz, ${ }^{1}$ and Florian Schlagenhauf ${ }^{1,2}$ \\ ${ }^{1}$ Department of Psychiatry and Psychotherapy, Charité Universitätsmedizin, 10117 Berlin, Germany, ${ }^{2}$ Max Planck Institute for Human Cognitive and Brain \\ Sciences, 04103 Leipzig, Germany, ${ }^{3}$ Department of Neurology, Otto-von-Guericke University, 39106 Magdeburg, Germany, ${ }^{4}$ Institute of Neurophysiology, \\ Charité Universitätsmedizin, 10117 Berlin, Germany, ${ }^{5}$ Department of Psychiatry, Psychotherapy, and Psychosomatics, Medical School Brandenburg, 16816 \\ Neuruppin, Germany, and 'Institute of Cognitive Neuroscience, University College London, WC1N 3AR London, United Kingdom
}

The striatum is known to play a key role in reinforcement learning, specifically in the encoding of teaching signals such as reward prediction errors (RPEs). It has been proposed that aberrant salience attribution is associated with impaired coding of RPE and heightened dopamine turnover in the striatum, and might be linked to the development of psychotic symptoms. However, the relationship of aberrant salience attribution, RPE coding, and dopamine synthesis capacity has not been directly investigated. Here we assessed the association between a behavioral measure of aberrant salience attribution, the salience attribution test, to neural correlates of RPEs measured via functional magnetic resonance imaging while healthy participants $(n=58)$ performed an instrumental learning task. A subset of participants $(n=27)$ also underwent positron emission tomography with the radiotracer $\left[{ }^{18} \mathrm{~F}\right]$ fluoro-L-DOPA to quantify striatal presynaptic dopamine synthesis capacity. Individual variability in aberrant salience measures related negatively to ventral striatal and prefrontal RPE signals and in an exploratory analysis was found to be positively associated with ventral striatal presynaptic dopamine levels. These data provide the first evidence for a specific link between the constructs of aberrant salience attribution, reduced RPE processing, and potentially increased presynaptic dopamine function.

Key words: aberrant salience; dopamine; orbitofrontal cortex; prediction error; reinforcement learning; ventral striatum

\section{Introduction}

Aberrant attribution of salience, i.e., to irrelevant or otherwise neutral stimuli, has been proposed as the central mechanism in the development of psychotic experiences (Heinz, 2002; Kapur, 2003). It has been suggested that aberrant salience attribution may arise from elevated dopaminergic neurotransmission (Heinz and Schlagenhauf, 2010; Winton-Brown et al., 2014), consistent with the replicated finding of heightened presynaptic dopamine levels in schizophrenia and individuals at risk of psychosis (Howes and Kapur, 2009; Howes et al., 2012) and with data from animal research connecting dopamine levels and models of salience attribution (Bay-Richter et al., 2009; Weiner and Arad, 2009; O'Callaghan et al., 2014).

\footnotetext{
Received Feb. 28, 2015; revised May 3, 2015; accepted May 26, 2015.

Author contributions: R.B., L.D., T.K., A.P., J.B., R.Bu., J.P.R., A.H., and F.S. designed research; R.Bo., L.D., T.G., T.K., A.P., and R.Bu. performed research; R.Bo., T.K., A.P., and F.S. analyzed data; R.Bo., L.D., T.G., J.B., J.P.R., A.H., and F.S. wrote the paper.

This work was supported by grants from Deutsche Forschungsgemeinschaft (SCHL1969/1-1, GRK-1123). We thank Yu Fukuda, Saineb Alaa-Eddine, Sarah Diner, and Jakob Kaminski for assistance during data acquisition.

The authors declare no competing financial interests.

Correspondence should be addressed to Rebecca Boehme, Department of Psychiatry and Psychotherapy, Charité Universitätsmedizin Berlin, Campus Mitte, Charitéplatz 1, 10117 Berlin, Germany. E-mail: rebecca.boehme@charite.de.

DOI:10.1523/JNEUROSCI.0805-15.2015

Copyright $\odot 2015$ the authors $\quad 0270-6474 / 15 / 3510103-09 \$ 15.00 / 0$
}

In healthy individuals, dopamine plays a key role in signaling incentive salience (Robinson and Berridge, 1993) and correlates with a teaching signal central in theories of reinforcement learning (Sutton and Barto, 1998). Teaching signals help adapt to an ever-changing environment. Phasic firing of midbrain dopamine neurons was demonstrated to encode the difference between received outcome and expected value (Montague et al., 1996; Schultz, 1997), termed the reward prediction error (RPE). These findings were mirrored in humans using fMRI: coding of RPEs in areas innervated by dopaminergic neurons was repeatedly shown, particularly in the striatum (O'Doherty et al., 2003; D'Ardenne et al., 2008). In schizophrenia, attenuated striatal RPE signals were observed (Murray et al., 2008; Gradin et al., 2011; Deserno et al., 2013; Schlagenhauf et al., 2014). An association of such signals with delusion severity was reported (Corlett et al., 2007; Schlagenhauf et al., 2009; Romaniuk et al., 2010) and attenuated striatal RPE signals were associated with self-reported delusion-like beliefs in healthy participants (Corlett and Fletcher, 2012). In a similar population, high levels of aberrant salience have been reported (Roiser et al., 2013) suggesting that there might be an association of the constructs of RPE and aberrant salience.

Recently, a paradigm was designed to measure the degree of aberrant salience-the salience attribution test (SAT; Roiser et 
al., 2009). Participants had to respond to different stimuli, some of which predicted reward while others were uninformative. Aberrant salience was operationalized as implicit (the speeding of responses to irrelevant stimulus features) and explicit measures (subjective probability ratings about reward contingencies). The implicit measure had the highest construct validity in healthy volunteers (Schmidt and Roiser, 2009). So far, it has been demonstrated that schizophrenia patients with delusions exhibited higher explicit aberrant salience than patients without (Roiser et al., 2009) as did individuals in an at-risk state for psychosis compared with healthy volunteers (Roiser et al., 2013). We found significantly higher implicit aberrant salience in a large sample of schizophrenia patients (A. Pankow, T. Katthagen, S. Diner, L. Deserno, R. Boehme, T. Gleich, M. Gaebler, H., A. Heinz, F., unpublished observations). fMRI results showed that the ventral striatum (VS) was activated by task-adaptive reward prediction during the SAT, while aberrant reward prediction responses have been observed inconsistently in the VS (Roiser et al., 2010, 2013).

Although there is an influential theoretical background motivating the idea that aberrant salience attribution may relate to weakened learning signals in the VS elicited by task-relevant cues (Heinz, 2002; Kapur, 2003; Corlett et al., 2007; Schlagenhauf et al., 2009; Heinz and Schlagenhauf, 2010; Winton-Brown et al., 2014), the association between these processes has yet to be investigated directly. Here, we related a behavioral measure of aberrant salience attribution derived from the SAT to RPEs assessed by fMRI during operant learning in a large sample of healthy participants. We hypothesized that high levels of aberrant salience attribution are associated with decreased VS RPE signals; we also investigated whether aberrant salience levels are associated with prefrontal RPE encoding. A subgroup underwent $\left[{ }^{18} \mathrm{~F}\right]$ fluoro-L-DOPA (FDOPA) PET to measure presynaptic striatal dopamine levels. Based on the proposal that aberrant salience attribution is accompanied by elevated presynaptic dopamine levels (Howes et al., 2012), we predicted a positive association with aberrant salience scores.

\section{Materials and Methods}

Participants. Fifty-eight healthy volunteers (23 female; mean age: $25.8 \pm$ 5.9 years, range 18-43) were included; participants were recruited through university mailing lists and from the institute subject database. They were free of Axis I psychiatric disorders, with no history of psychiatric disorders in first-degree relatives, and no current or past alcohol or substance abuse (other than nicotine; First et al., 2001). There were three separate appointments for the SAT, fMRI, and PET measurements. Peter's Delusion Inventory (PDI; Peters et al., 1999) and Schizotypy Personality Questionnaire (SPQ; Raine, 1991) were used to approximate psychosis-like experiences. The local ethics committee approved the study and written informed consent was obtained after complete study description. A subgroup of this sample also performed a different behavioral task during fMRI, published separately (Deserno et al., 2015).

Aberrant salience task. The SAT was used to measure aberrant salience behaviorally. A more detailed description is provided in the original publication (Roiser et al., 2009). In short, a stimulus appeared on the screen as a cue, which could vary across two dimensions: color (red or blue) and form (animal or household object; Fig. 1). While the stimulus features on one dimension predicted reward availability (e.g., red vs blue: 87.5 vs $12.5 \%$ ), the other dimension carried no predictive information about the occurrence of reward and was therefore irrelevant (e.g., $50 \%$ reward for both animal and household features). Right after the cue, participants had to respond to the presentation of a square (the probe) to win money. They were instructed that faster responses yielded higher rewards, but reward was not always available. If the trial was not reinforced, the message "Sorry-no money available" was displayed after the probe disappeared. If reinforced, "hit" responses (made before the probe

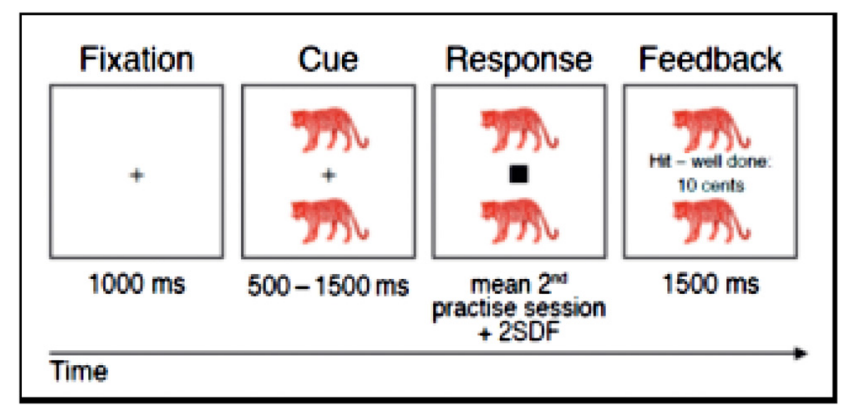

Figure 1. One trial of the SAT. Participants had to respond to a probe preceded by stimuli that varied in two dimensions (color, shape), only one of which was relevant for predicting reward ( 80 vs $20 \%$ ). The other dimension carried no predictive information ( $50 \%$ win probability). Probe duration was calculated from individual reaction times in the second practice session [mean \pm 2 SDs from the fastest half of trials (SDF)]. Faster responses yielded higher rewards, about which feedback was given following each trial.

disappeared) that were slower than the participant's own mean reaction time (RT) (measured during an earlier practice session) resulted in the message "Hit-good: 10 cents." For hit responses faster than the participant's mean practice RT the following messages appeared: "Quickvery good: X cents" (for responses up to 1.5 SDs above their mean RT) and "Very quick-excellent: X cents" (for responses faster than 1.5 SD). The scaling of reward in each trial was calculated using the equation: $\mathrm{X}=$ $10+45 \times($ mean RT $-\operatorname{trial} \mathrm{RT}) /(3 \times \mathrm{SD})$, up to a maximum of 40 cents per trial. Implicit aberrant salience attribution was measured through RTs and calculated as follows: subtraction of the RTs to subjective low reward stimuli from the RTs to subjective high reward stimuli followed by square root transformation to reduce skew. Participants performed the task in two separate blocks of equal length, over which values were averaged. We used this implicit measure of aberrant salience, which has been shown to have a high construct validity (Schmidt and Roiser, 2009), to create two groups of 29 subjects each ("high" and "low") via a median split. Additionally, we explored the robustness of the results using an extreme group approach with two groups of 20 people each including the highest and lowest values of implicit aberrant salience attribution. In addition to the implicit measure, we also obtained the explicit measure, based on an individual rating after each of the two blocks. Participants rated the individual stimuli on a visual analog scale by estimating how often each of the four stimulus types had been reinforced $(0-100)$. To analyze the development of salience attribution over the first block of the experiment, RTs were centered using the mean RT from the practice session. RTs were separated into high and low predictive probability trials and irrelevant trials. The latter were categorized as subjective good or bad (based on mean RT/based on individual ratings). RTs were averaged over time bins of five trials, resulting in six data points.

Operant learning task. For operant learning, a probabilistic reinforcement learning paradigm was used. Participants were instructed to choose one of two different stimuli appearing simultaneously on the screen during a decision window of $1.5 \mathrm{~s}$. Stimuli were randomly assigned to the left or right side (Fig. 2A). If no response occurred within the decision window, the message "too slow!" was displayed and the next trial began. One hundred and sixty trials were administered. Each trial consisted of stimulus presentation $(1.5 \mathrm{~s})$; feedback $(0.5 \mathrm{~s})$; and a jittered, exponentially distributed intertrial interval ( $\min 1 \mathrm{~s}, \max 12.5 \mathrm{~s}$ ). On each trial, participants could win or lose money. They were instructed to try to win as much money as possible. One symbol was associated with a high reward probability $(80 \%)$ and a low loss probability $(20 \%)$, and the inversed probabilities were assigned to the other symbol. This probability distribution switched at various points in the experiment: at the beginning and end were stable periods without switching (first block: 55 trials; last block: 35 trials); in the middle, shorter blocks alternated between 15 and 20 trials (Fig. 2B). Feedback for win trials consisted of the message "Win! +10 Cent" and for lose trials of the message "Loss! -10 Cent." Each participant practiced the task during a training session without reversals of reward contingencies, but was notified that there might be switches 
A

cue

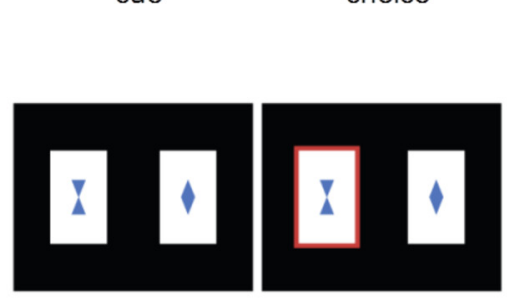

B

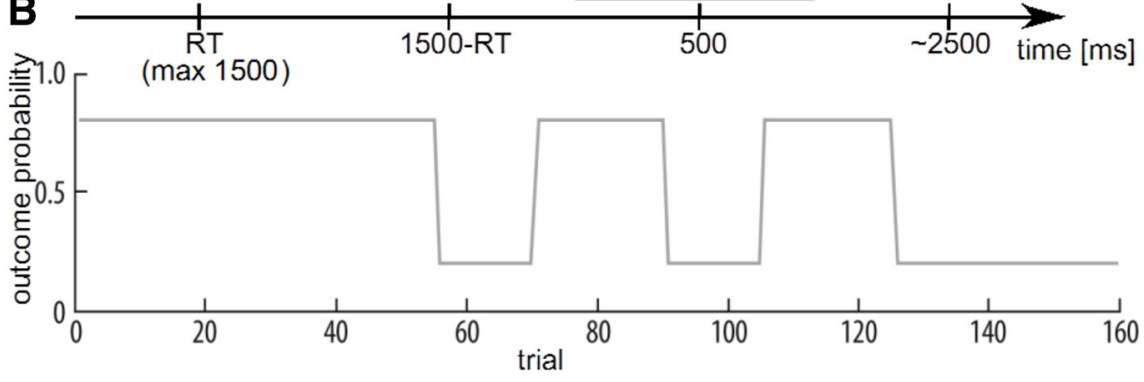

outcome ITI


I.⿴囗十
政

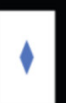
.

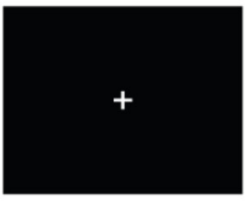
(1)



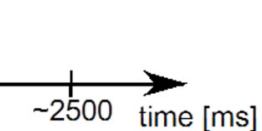

Figure 2. Operant learning task. A, Participants had to choose the symbol with the higher reward probability to win money. $\boldsymbol{B}$, Reward probability associated with one of the symbols changed over the course of the whole experiment (the other symbol's reward probability was the inverse).

during the main experiment. A minimum of $3 €$ and a maximum of $10 €$ could be won on the task. Behavioral performance was quantified as the percentage of "correct" responses, i.e., choosing the currently better symbol. Groups were compared using two sample $t$ tests. Behavioral data were analyzed using SPSS 19.

Reinforcement learning model. A reinforcement learning model was applied to the operant learning task to quantify learning dynamically and to generate trial-by-trial RPEs for each individual to serve as regressors in the fMRI analysis. We used a Q-learning algorithm, which estimates five free parameters for every participant to model their choices during the reinforcement learning paradigm. The algorithm tracks the expected outcome value (the "Q-value") of the chosen stimulus $a$ (Sutton and Barto, 1998) on each trial $t$. This expected value was adjusted according to the RPE $\delta_{Q a, t}$, which is defined as the difference between the received outcome $R_{t}$ and the expected outcome $Q_{a, t}$ for the chosen stimulus:

$$
\delta_{Q a, t}=R_{t}-Q_{a, t},
$$

$R_{t}$ denotes two separate free parameters: for rewarded and punished trials. Instead of setting $R_{t}$ for reward and punishment to fixed values ( 1 and -1 ), we allowed them to vary individually as free parameters to capture variation in reward and punishment sensitivity (Schlagenhauf et al., 2014). The RPE is used as a teaching signal to update expected values iteratively trial by trial:

$$
Q_{a, t+1}=Q_{a, t}+\alpha \delta_{Q a, t} .
$$

Here, $\alpha$ determines the learning rate, which also was estimated separately for reward and punishment outcomes. The learning rate describes how quickly expectations change with respect to the current RPE. There was another free parameter $Q_{i}$ that specified the initial $Q$-values for one option (a bias to choose one or the other stimulus initially; Schlagenhauf et al., 2014). A softmax rule was used to estimate the probability of choices $\left(p_{a}(t)\right)$ based on expected values:

$$
p_{a}(t)=\frac{\exp \left(Q_{a}(t)\right)}{\exp \left(Q_{a}(t)\right)+\exp \left(Q_{b}(t)\right)} .
$$

Here, the used softmax equation does not contain a free temperature parameter, because reward and punishment sensitivities cover the same behavioral variation and render the temperature redundant. Parameters of the model were estimated by applying expectation maximization with empirical priors and the model evidence was approximated by integrating out the free parameters over the likelihood via sampling from the prior distribution (Huys et al., 2011, 2012). For between-group comparison, all five parameters, i.e., reward and punishment learning rates, reward and punishment sensitivities, and initial Q-value, were entered into a multivariate ANOVA (MANOVA).

Only subjects whose choice behavior was explained better than chance (based on the likelihood that the observed behavioral data are given by the parameters) were included in further modeling-based analysis (52 of 58 subjects: four from the low and two from the high aberrant salience group were excluded). Thus, time series derived from these parameters reflect important aspects of the behavioral data and can be regressed against imaging data in a meaningful way.

fMRI. A 3.0 tesla Siemens trio scanner with a 12-channel head coil was used to acquire 423 T2-weighted EPIs containing 40 slices (TR = $2090 \mathrm{~ms}, \mathrm{TE}=22 \mathrm{~ms}$, slice thickness $2.5 \mathrm{~mm}$, matrix size $64{ }^{*} 64$, field of view $4888^{\star} 488 \mathrm{~mm}^{2}$, in-plane voxel resolution $3 \mathrm{~mm}^{2}$, flip angle $=$ $\left.90^{\circ}\right)$. Field distortion maps and T1-weighted anatomical images were also acquired. fMRI data were analyzed using statistical parametric mapping (SPM8; Wellcome Department of Imaging Neuroscience, London, UK; http://www.fil.ion.ucl.ac.uk/spm) in MATLAB R2010b (The MathWorks). The following steps were performed: slice-time and motion correction including unwarping, coregistration of the mean EPI and the anatomical image, spatial normalization to the MNI T1 template, and segmentation of the T1 image using the unified segmentation approach (Ashburner and Friston, 2005). Normalization parameters were applied to all EPIs. Finally, all images were spatially smoothed with an isotropic Gaussian kernel of $6 \mathrm{~mm}$ full-width at half-maximum.

For statistical analysis of the BOLD response, the general linear model approach was used as implemented in SPM8. In an event-related design, feedback onsets were convolved with the hemodynamic response function and trial-by-trial RPEs derived from the learning model were added as a parametric modulator. One additional regressor marked trials where no answer occurred. To account for movement-associated variance, realignment parameters and their first temporal derivative of translational movement plus an additional regressor marking scans with $>1 \mathrm{~mm}$ scanto-scan movement were included as regressors of no interest. Individual contrast images were taken to a random effects group-level analysis (onesample $t$ tests for within-group and two-sample $t$ tests for between-group comparisons). To correct for multiple comparisons, statistics are reported using FWE correction at the voxel level across the whole brain. Based on our a priori hypothesis about the negative association between ventral striatal RPE signal and measures of aberrant salience, parameter estimates from the fMRI activations were extracted from a $2 \mathrm{~mm}$ sphere around within-group (over all subjects) peak voxels and subjected to a correlation analysis with aberrant salience measures derived from the SAT (one-tailed, based on our a priori hypothesis) using SPSS19. For group comparisons, the search volume was restricted to those voxels showing a significant main RPE effect at $p<0.05$ (whole-brain corrected) across the entire sample (Table 1 ).

PET. A subgroup of 27 subjects (mean age $28.2 \pm 6,12$ female) also underwent FDOPA PET. Data were acquired using a Philips Gemini TF16 time-of-flight PET/CT scanner in 3D mode. After a low-dose transmission CT scan, a 60 min dynamic 3D "list-mode" emission recording started after intravenous bolus administration of $200 \mathrm{MBq}$ FDOPA. The following steps were performed: CT-based tissue attenuation and scatter correction, reconstruction (OSEM, 16 iterations, six subsets) and framing ( 30 frames: $3 \times 20 \mathrm{~s}, 3 \times 1 \mathrm{~min}, 3 \times 2 \mathrm{~min}, 3 \times 3 \mathrm{~min}, 7 \times 5 \mathrm{~min}, 1 \times$ 
Table 1. Prediction error-related activation

\begin{tabular}{|c|c|c|c|c|c|c|c|}
\hline Anatomical region & $p$ (FWE-corr.) & $T$ & $Z$ & $x$ & $y$ & $z$ & Cluster size \\
\hline \multirow[t]{2}{*}{ Ventral striatum } & $<0.001$ & 7.14 & 5.94 & 14 & 10 & -10 & 55 \\
\hline & 0.021 & 5.83 & 5.08 & -10 & 12 & -10 & 11 \\
\hline \multirow[t]{2}{*}{ Putamen } & 0.002 & 6.69 & 5.64 & 24 & -12 & 8 & 58 \\
\hline & 0.017 & 5.93 & 5.15 & -30 & -14 & -2 & 13 \\
\hline Caudate & 0.002 & 6.59 & 5.58 & 20 & 0 & 22 & 23 \\
\hline \multirow[t]{2}{*}{ Superior frontal gyrus } & 0.004 & 6.42 & 5.47 & -36 & 46 & -12 & 21 \\
\hline & 0.004 & 6.41 & 5.46 & -12 & 36 & 50 & 18 \\
\hline \multirow[t]{2}{*}{ Middle frontal gyrus } & 0.002 & 6.73 & 5.65 & -32 & 48 & -12 & 48 \\
\hline & 0.012 & 6.05 & 5.23 & 38 & 36 & -14 & 14 \\
\hline \multirow[t]{2}{*}{ Inferior frontal gyrus } & 0.002 & 6.67 & 5.63 & -52 & 38 & 8 & 94 \\
\hline & 0.003 & 6.52 & 5.53 & -40 & 28 & -16 & 12 \\
\hline \multirow[t]{2}{*}{ Medial frontal gyrus } & 0.003 & 6.45 & 5.49 & 2 & 66 & 26 & 26 \\
\hline & 0.012 & 6.04 & 5.22 & 2 & 48 & 48 & 11 \\
\hline Anterior cingulate & 0.002 & 6.61 & 5.59 & 14 & 42 & 4 & 12 \\
\hline \multirow[t]{3}{*}{ Cingulate gyrus } & $<0.001$ & 7.78 & 6.29 & 0 & 0 & 36 & 52 \\
\hline & 0.004 & 6.36 & 5.43 & -2 & -30 & 38 & 19 \\
\hline & 0.004 & 6.39 & 5.45 & 4 & -40 & 36 & 41 \\
\hline Inferior parietal lobule & 0.002 & 6.69 & 5.64 & 56 & -56 & 38 & 43 \\
\hline Superior temporal gyrus & 0.002 & 6.69 & 5.64 & 46 & -62 & 28 & 28 \\
\hline Middle temporal gyrus & 0.007 & 6.22 & 5.34 & -64 & -50 & -6 & 11 \\
\hline Fusiform gyrus & 0.003 & 6.43 & 5.48 & 30 & -82 & -20 & 18 \\
\hline Thalamus & 0.008 & 6.15 & 5.30 & 20 & -14 & 20 & 12 \\
\hline Parahippocampal gyrus & 0.012 & 6.04 & 5.22 & -24 & -6 & -16 & 13 \\
\hline \multirow[t]{5}{*}{ Cerebellum } & $<0.001$ & 7.29 & 6.01 & -36 & -82 & -28 & 80 \\
\hline & 0.002 & 6.58 & 5.57 & 24 & -70 & -34 & 14 \\
\hline & 0.004 & 6.39 & 5.45 & -14 & -52 & -14 & 12 \\
\hline & 0.004 & 6.35 & 5.43 & -16 & -84 & -30 & 37 \\
\hline & 0.006 & 6.27 & 5.37 & -2 & -62 & -30 & 14 \\
\hline
\end{tabular}

One-sample $t$ test over all participants, at $p<0.05$ FWE corrected across the whole brain, cluster size $>10$.

Table 2. Development of RTs over the course of the first block

\begin{tabular}{|c|c|c|c|c|c|c|}
\hline & Time bin 1 & Time bin 2 & Time bin 3 & Time bin 4 & Time bin 5 & Time bin 6 \\
\hline Relevant high & 8.7938 & 5.3766 & 4.2249 & 1.8835 & 1.9579 & -4.7346 \\
\hline Relevant low & 10.9938 & 9.7456 & 13.38 & 9.2145 & 7.6214 & 8.3460 \\
\hline Irrelevant "good" (mean RT) & 10.9766 & 7.0214 & 2.9525 & -3.3958 & -3.5027 & -8.2518 \\
\hline Irrelevant "bad" (mean RT) & 14.7318 & 6.5662 & 15.8352 & 11.9456 & 10.4352 & 10.8318 \\
\hline Irrelevant "good" (rating) & 15.5456 & 4.5525 & 5.1835 & -0.3751 & 2.5938 & -0.9042 \\
\hline Irrelevant "bad" (rating) & 10.1628 & 9.0352 & 13.6042 & 8.9249 & 4.3387 & 3.1552 \\
\hline
\end{tabular}

In milliseconds, relative to the mean practice RT. Trial time bins include five trials each, mean over all participants. For the irrelevant dimension subjective "good" and "bad" are reported as defined relative to the mean RT over the block and relative to the subjective rating at the end of a block.

6 min) of list-mode data, coregistration of a mean emission image and the individual T1 image, and spatial normalization of the T1 image using the unified segmentation approach (Ashburner and Friston, 2005). Normalization parameters were applied to all frames. Presynaptic dopamine synthesis capacity was quantified as FDOPA $K_{i}\left(\min ^{\times 1}\right)$ voxel by voxel. $K_{i}$ was estimated using the frames from 20 to $60 \mathrm{~min}$ of the recording by applying Gjedde-Patlak linear graphical analysis (Patlak and Blasberg, 1985). Values from a standard cerebellum mask (excluding Vermis, WFU PickAtlas) were used as input function. $K_{i}$ values were extracted after normalization to MNI space from four bilateral ROIs: from limbic (ventral), sensorimotor, and associative striatum (Martinez et al., 2003; Howes et al., 2012) and additionally from $2 \mathrm{~mm}$ spheres around the peak voxel of ventral striatal RPE-associated activation obtained in the fMRI analysis over all participants. $K_{i}$ values were compared between high and low aberrant salience groups using a MANOVA and correlated with aberrant salience scores using Pearson correlations within SPSS19. Correlations were corrected for multiple comparisons via Bonferroni corrections.

\section{Results}

\section{Behavior during the SAT}

The implicit measure of aberrant salience (AbSal) had a mean of $3.1 \pm 1.1$ (range $0.7-6.55$ ). Compared with previously published data, this sample displays a rather small overall range (values before transformation for comparability: $11.6 \pm 7.8 \mathrm{~ms}$; see Roiser et al., 2009, where the healthy sample was found around $16.5 \pm 10.3$ and 14.5 \pm 14.9 , Roiser et al., 2013: $24.6 \pm 21.9 \mathrm{~ms}$, and Roiser et al., 2010: $15.7 \pm 8.5 \mathrm{~ms}$ ). During the first block of the experiment, RTs for the subjective "good" cue decreased relative to the subjective "bad" cue (Table 2), indicating that AbSal attribution was acquired during the experiment and not based on a pre-existing bias.

On the basis of the AbSal measure, two groups of 29 participants each were formed via a median split. The low AbSal group had a mean score of $2.27 \pm 0.56$ (range 0.7-3.06), and the high AbSal group had a mean score of $3.9 \pm 0.77$ (range 3.09-6.55; for more details see Table 3$)$. These two groups did not differ in age $\left(t_{(55)}=1.03, p=0.32\right)$, gender $\left[\chi^{2}(1, N=58)=0.29, p=0.9\right]$, or verbal intelligence $\left(t_{(54)}<0.001, p>0.99\right)$. There was no difference in the used measures of delusion-like experiences (PDI: $t_{(56)}=0.86, p=0.4$, mean: low AbSal $=6.7 \pm 5.7$, high AbSal $=$ $7.5 \pm 5.6$, overall range $0-17$; SPQ: $t_{(45)}=0.7, p=0.48$, mean: low $\mathrm{AbSal}=18.35 \pm 16$, high AbSal $=15.5 \pm 11.5$, overall range $1-73)$ or in the measure of adaptive salience, i.e., reaction time 
Table 3. Overview modeling parameters and aberrant salience attribution for low and high aberrant salience groups

\begin{tabular}{lcc}
\hline Parameter & Low AbSal group & High AbSal group \\
\hline Rrew & $3.1 \pm 1.5$ & $3.5 \pm 1.2$ \\
Rpun & $-0.97 \pm 0.35$ & $-0.94 \pm 0.29$ \\
$\alpha_{\text {rew }}$ & $0.68 \pm 0.13$ & $0.57 \pm 0.19$ \\
$\alpha_{\text {pun }}$ & $0.61 \pm 0.08$ & $0.63 \pm 0.1$ \\
$Q$ & $-0.14 \pm 0.27$ & $-0.18 \pm 0.3$ \\
AbSal block 1 (ms) & $5.9 \pm 5.5$ & $15 \pm 7.8$ \\
AbSal block 2 (ms) & $6.7 \pm 5.5$ & $19 \pm 14$ \\
AbSal mean (ms) & $6.3 \pm 3$ & $17 \pm 7.6$ \\
\hline
\end{tabular}

Rrew, reward sensitivity; Rpun, punishment sensitivity; $\alpha_{\text {rew }}$, reward learning rate; $\alpha_{\text {pun }}$, punishment learning rate; $Q$, initial $Q$ value; AbSal, implicit aberrant salience (RTs); all \pm SD.

differences between the cues, which actually predicted reward probabilities $(t=-0.93, p=0.4)$.

Mean AbSal values in the extreme groups were $2 \pm 0.5$ for the low AbSal group and $4.3 \pm 0.7$ for the high AbSal group. There was a significant difference on the PDI measure for the two extreme groups: $t=2.5, p=0.018$ (mean: low AbSal groups $=4 \pm$ 5 , high AbSal group $=8.9 \pm 4.9$ )

\section{Behavior during the operant task}

On the operant learning task, performance of the two groups did not differ significantly: mean percentage correct responses were $74.75 \pm 11.5 \%$ for the low AbSal and $76.7 \pm 7.3 \%$ for the high AbSal group $\left(t_{(56)}=0.77, p=0.44\right)$.

With respect to the modeling analysis, a parameter $\times$ group MANOVA revealed a significant group difference only for reward learning rate $\alpha$ (mean $\alpha$ : low AbSal group $=0.68 \pm 0.13$, high AbSal group $=0.57 \pm 0.19, F_{(1,57)}=7.62, p=0.0078$; an overview over model parameters is given in Table 3$)$. After exclusion of the six participants whose behavior was not explained better than chance by the model, this effect remained significant $\left(F_{(1,51)}=5.58, p=0.02\right)$. The likelihood of the observed choice data was not related to AbSal scores.

The extreme group approach rendered the effect of the learning rate difference nonsignificant $\left(t_{(40)}=1.2, p=0.25\right)$.

\section{Neural correlates of RPEs}

RPEs covaried with BOLD response in a frontostriatal network including VS, cingulate cortex, parietal and temporal cortex, medial prefrontal cortex, orbitofrontal cortex (OFC), hippocampus, and cerebellum, which survived whole-brain FWE correction (FWE-WB; see Table 1).

The difference between the high compared with the low AbSal group in RPE-related activation extracted at the peak coordinate in the VS (right: $[1410-10], Z=5.94$, $\mathrm{P}_{\text {FWE-WB }}<0.001$; left: $\left.[-1012-10], Z=5.09, \mathrm{P}_{\mathrm{FWE}-\mathrm{WB}}=0.021\right)$ was significant on the left $\left[t_{(50)}=1.8, p=0.04\right.$ (one-tailed) $]$ and showed a trend toward significance on the right $\left[t_{(50)}=1.54, p=0.063\right.$ (one-tailed) $]$, indicating an association of higher levels of AbSal with reduced RPE signaling in the VS. When correlated over both groups, AbSal and RPE activation showed a negative association in the right VS $\left[r=-0.23, p=0.045\right.$ (one-tailed), $R^{2}=0.05$; Fig. 3$]$ and also approached significance in the left VS $[r=-0.2, p=0.08$ (onetailed), $\left.R^{2}=0.04\right]$.

The extreme group approach rendered this finding more significant [right VS: $t_{(40)}=2.044, p=0.049$ (two-tailed), left VS: $t_{(40)}=1.4, p=0.17$ (two-tailed)].

In addition to our a priori hypothesis with respect to the VS, we performed a voxelwise analysis (restricted to regions showing a main effect of RPE, as above), which revealed a

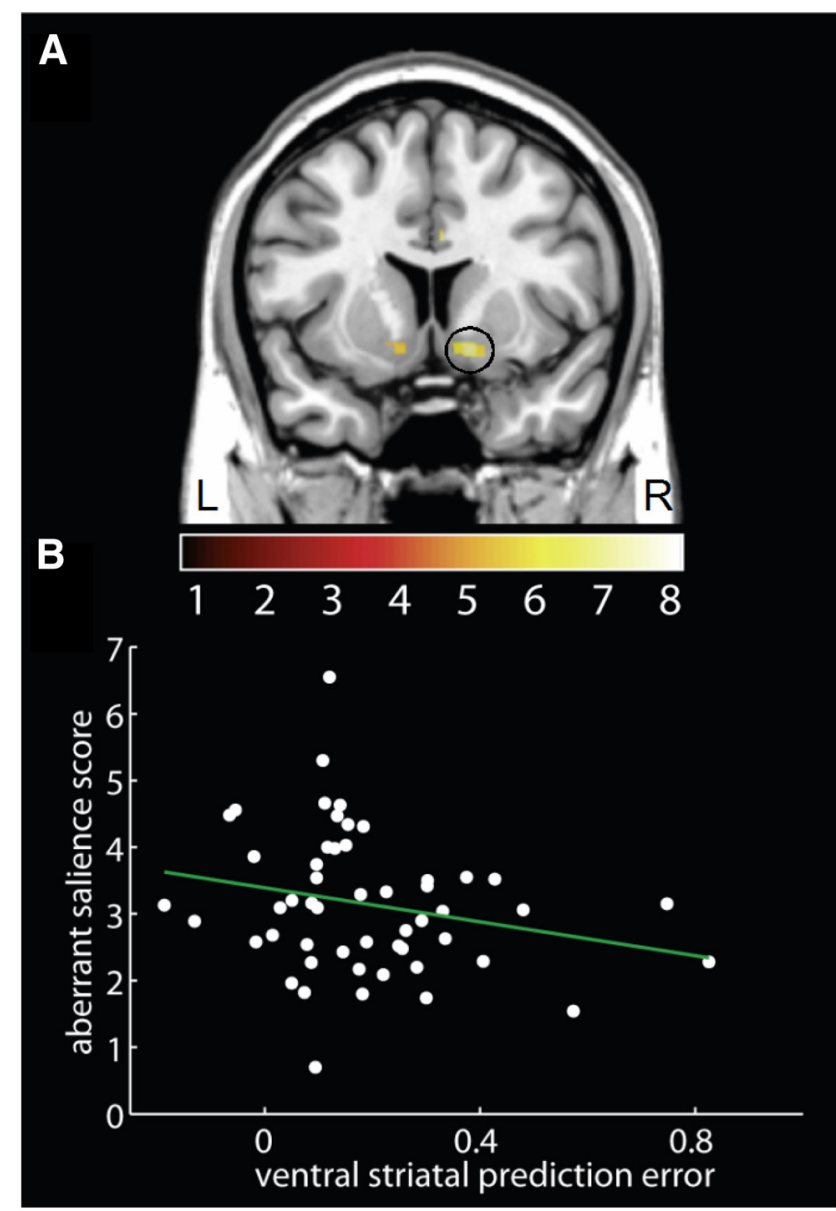

Figure 3. Ventral striatal prediction error signals correlate negatively with aberrant salience scores. $\boldsymbol{A}$, Prediction error-associated activation in the striatum $(y=10)$. The image is thresholded at $p<0.05$ (FWE-WB) and the color bar indicates $t$ values. $\boldsymbol{B}$, Correlation of parameter estimates from the right ventral striatum [14 $10-10]$ with aberrant salience scores $[r=$ $-0.23, p<0.05$ (one-tailed), $\left.R^{2}=0.05\right]$. L, left; $R$, right.

significant group difference in the left OFC $(Z=4.52,[-30$ $46-14], \mathrm{P}_{\text {SVC main effect }}=0.003$; Fig. $4 A$ ): while participants in the low AbSal group encoded RPEs in this region, this signal was reduced in the high AbSal group (Fig. 4B).

This finding was found to be robust using the two extreme AbSal groups $\left(Z=3.99, \mathrm{P}_{\text {SVC main effect }}=0.023\right)$.

Presynaptic dopamine levels and aberrant salience measures To relate presynaptic dopamine levels $\left(K_{i}\right)$ to aberrant salience attribution, a subsample of 27 subjects also underwent FDOPA PET (low AbSal: $n=14$, mean AbSal score $=2.2 \pm 0.38$, high AbSal $n=13$, mean AbSal score $=3.9 \pm 1.05)$. These AbSal subgroups did not differ in any of the demographic parameters $(t$ values $<1.1, p$ values $>0.26$ ). There was no significant difference in $K_{i}$ levels between the AbSal groups in the limbic, associated, or sensorimotor striatum $(t$ values $<1.5, p$ values $>0.16)$. There was no correlation between $K_{i}$ values in ROIs of striatal subregions (limbic, associative, and sensorimotor) and the implicit aberrant salience score ( $r$ values $<0.25, p$ values $>0.2$ ). In an exploratory analysis, $K_{i}$ values were extracted from a sphere around the peak voxels of RPE signals in the VS. These were positively correlated with aberrant salience measures in the right VS $(r=0.54, p=$ $0.004, R^{2}=0.29$; Fig. 5), while no association was found in the left VS $\left(r=0.19, p=0.35, R^{2}=0.036\right)$. No significant correla- 


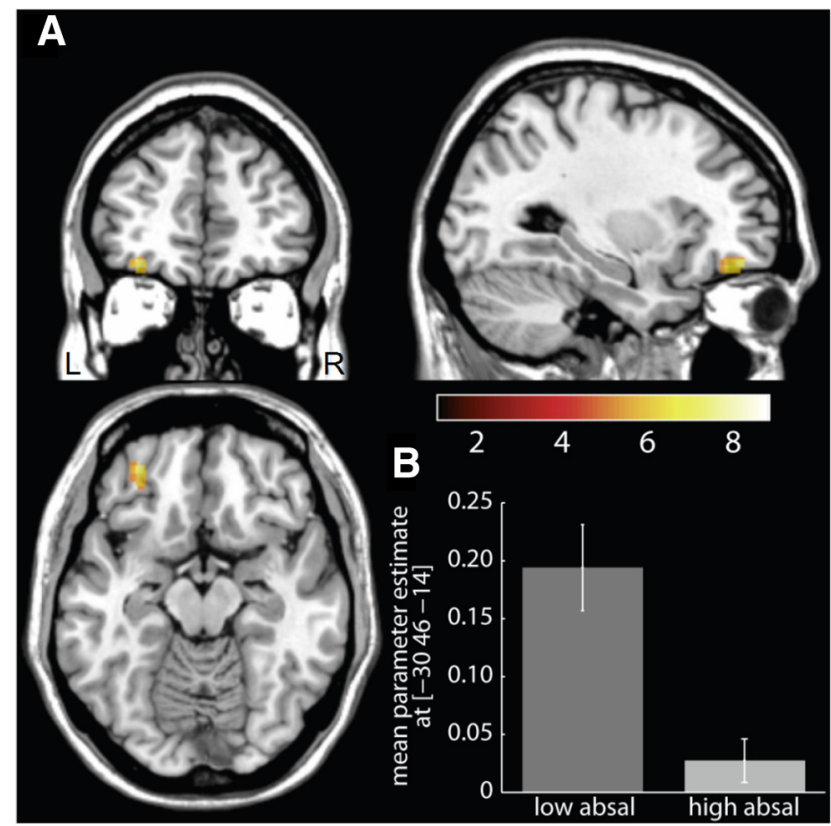

Figure 4. Low and high AbSal groups differ in prediction error-associated activation in the left orbitofrontal cortex. $\boldsymbol{A}$, Contrast between low and high aberrant salience groups, displayed at $p<0.001$ (uncorrected). $\boldsymbol{B}$, Mean parameter estimates for the groups of low and high aberrant salience at [ $-3046-14]$. Error bars indicate SEM and the color bar indicates $t$ values. $L$, left; $R$, right.

tion was observed of RPE BOLD signal with $K_{i}$ in this ROI $(p=0.89)$.

\section{Explicit aberrant salience}

Following the suggestion of one of our reviewers we also explored associations with the explicit measure of aberrant salience $($ mean $=2.2 \pm 1.4$, range $=0-5.5)$. This measure was not correlated with the implicit aberrant salience measure $(r=-0.18$, $p=0.18)$ and there were no relationships of this measure with fMRI ( $r$ values $<0.09, p$ values $>0.5)$ or PET-derived values $(r$ values $<0.15$, $p$ values $>0.43)$.

\section{Discussion}

Here we present first evidence for an association of aberrant salience attribution derived from the SAT (Roiser et al., 2009) with reinforcement learning signals and neurochemical measures of presynaptic dopamine in a sample of healthy controls: (1) participants with higher levels of aberrant salience attribution displayed reduced coding of RPEs in VS and OFC during instrumental learning and (2) aberrant salience attribution correlated positively with ventral striatal presynaptic dopamine levels measured in the area activated by RPEs. These results provide first proof that interindividual differences in a measure of aberrant salience attribution relate to behavioral and neural correlates of reinforcement learning and measures of dopaminergic neurotransmission.

Activation of the VS in response to RPEs has been repeatedly demonstrated (O'Doherty et al., 2003; D'Ardenne et al., 2008); these learning signals are important for trial-by-trial updating of reward expectations and underlie behavioral adaptation (Kehagia et al., 2010; Boureau and Dayan, 2011; Deserno et al., 2013; Ullsperger et al., 2014). Here we show that coding of such adaptive learning signals is relatively lower in subjects displaying higher aberrant salience attribution.

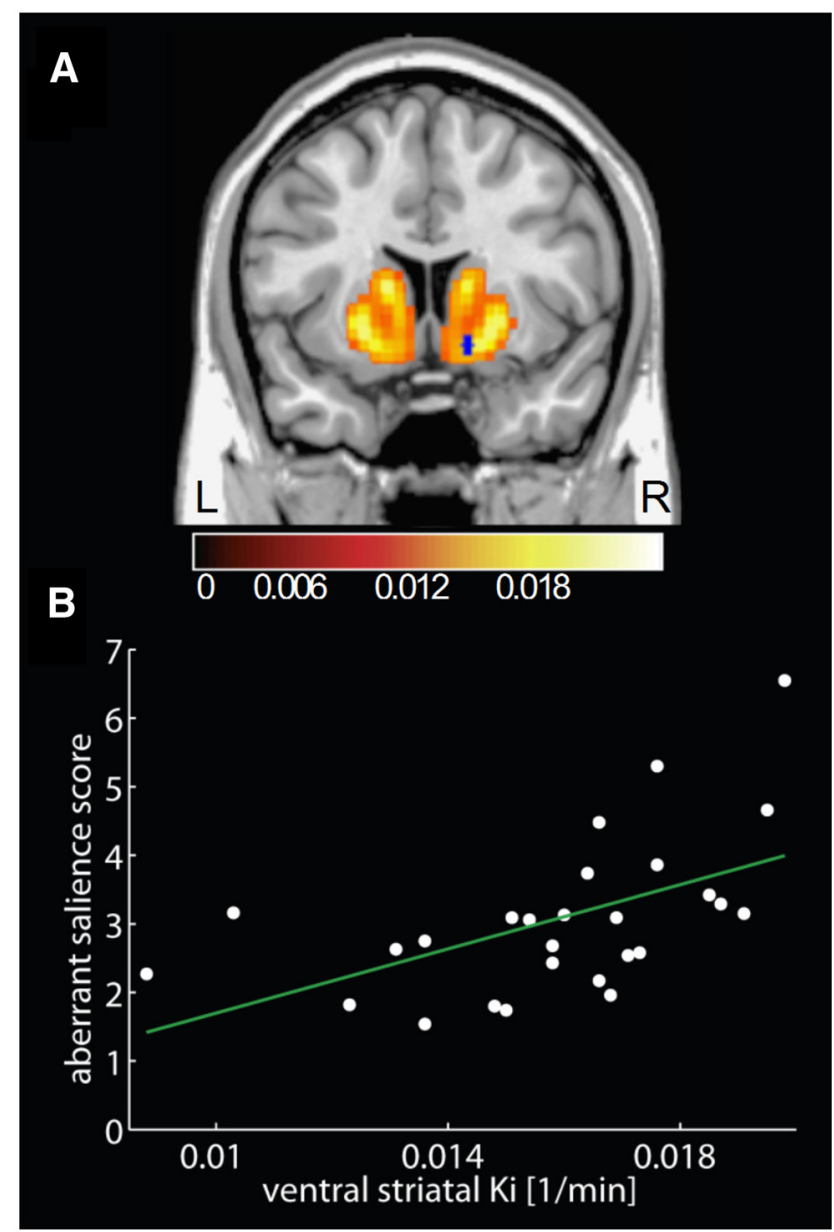

Figure 5. PET $K_{i}$ values (indicated by the color bar) quantifying presynaptic dopamine levels correlate positively with aberrant salience scores. $A$, PET $K_{i}$ at $y=10$, mean over all participants. $\boldsymbol{B}$, Correlation of aberrant salience scores with $K_{i}$ values extracted from peak coordinate of the prediction error-related activation in the right ventral striatum ([14 10 - 10], indicated in blue in $A ; r=0.54, p<0.01, R^{2}=0.29$ ). L, left; $\mathrm{R}$, right.

The finding of a negative correlation between implicit aberrant salience attribution and VS RPEs, here in healthy controls, is in line with studies showing that people with psychotic experiences show reduced RPE signals (Corlett et al., 2007; Schlagenhauf et al., 2009; Romaniuk et al., 2010; Corlett and Fletcher, 2012). Note, though, that we only obtained an association with one self-report scale of delusion-like experiences in the extreme group approach. This does not necessarily contradict the continuum model of psychosis, because aberrant salience measures of our healthy sample covered the lower range of possible aberrant salience values, which might have been insufficient to detect an association with psychopathology (Roiser et al., 2009, 2010, 2013). The RT measure might be too fine-grained to correlate with the coarser self-assessment scales. It is also conceivable that a number of factors have to interact to lead to psychosis-like experiences; aberrant salience attribution might be only one of them.

Alternatively, high aberrant salience participants might engage in a different strategy to solve the task, which might lead to reduced fMRI signals (Schönberg et al., 2007). We cannot make any claims about possible group differences here, because we did not compare behavioral strategies. Instead we used one computational model for all participants to estimate meaningful regressors. Both subgroups were well explained by the learning model 
and did not differ regarding their reward or punishment sensitivity. However, the finding of a lower learning rate in the group with higher aberrant salience measures indicates slower updating after a reward. Decreased reward learning rates might lead to slower updating of the conditioned response after an aberrant relationship between expectations and irrelevant cues was established initially. To some extent our presented findings challenge the notion that aberrant salience and flexible reward-based learning represent independent constructs. If the aberrant salience measure constitutes a different conceptualization of the same mechanism evaluated by RPEs, aberrant salience would simply reflect altered RPE-based learning. In this case, correlating these measures would be redundant, but the use of reinforcement learning paradigms for investigations of the aberrant salience hypothesis in psychotic patients would be further supported. On a biological level, there is also evidence for independent dopaminergic processing pathways of RPEs and salience: from studies in monkeys the proposition arises that different subpopulations of dopaminergic neurons encode RPEs and salience (Matsumoto and Hikosaka, 2009; Matsumoto and Takada, 2013). For future studies, pharmacological challenges of the dopamine system represent a powerful tool to proof conceptual overlap as well as independence of both constructs on a psychological and biological level. One first study approached this question recently in Parkinson's disease (Nagy et al., 2012).

Furthermore, we found first evidence that aberrant salience attribution was positively related to presynaptic dopamine levels in the same region of the VS that was activated by RPEs. Increased striatal dopamine levels were proposed to be involved in stochastic assignment of salience to irrelevant stimuli (Heinz, 2002; Kapur, 2003; Poletti et al., 2014). Elevated levels of striatal presynaptic dopamine synthesis capacity are a well established finding in schizophrenia patients (Howes et al., 2012), and have been observed in individuals in an at-risk mental state for psychosis (Egerton et al., 2013). Animal models and human PET imaging suggest an overactivation of the dopaminergic system as possible mechanism: because of altered regulatory processes the spontaneous activity of dopaminergic neurons might be increased (Abi-Dargham et al., 2000; Lodge and Grace, 2007; Goto and Grace, 2008; Mizrahi et al., 2012). A general upregulation of tonic activity, leading to heightened phasic responses, could in turn render all stimulus-driven inputs important contributing to aberrant salience attribution to irrelevant stimuli (Grace, 2012). In a similar vein, heightened aberrant salience attribution might be associated with decreased VS RPE BOLD signals because of stochastic VS activity leading to deflated covariation between RPE size and BOLD signal. Our data complement these lines of research and suggest that aberrant salience attribution is directly correlated with elevated dopamine synthesis capacity and reduced encoding of RPEs.

In the whole-brain analysis, we found decreased OFC activation in subjects with higher values of aberrant salience attribution. The OFC is associated with reward processing in animals (Roesch and Olson, 2004; Padoa-Schioppa and Assad, 2006) and was repeatedly implicated in the reward circuit in humans (Kringelbach and Rolls, 2004). Functional and anatomical abnormalities of this region are found in major psychiatric disorders (Jackowski et al., 2012), especially in obsessive compulsive disorder (Greenberg et al., 2000; Menzies et al., 2008), but also in schizophrenia (Meador-Woodruff et al., 1997; Malchow et al., 2015). Accumulating evidence indicates that the OFC contributes to updating stimulus-outcome associations by providing information about expectations to downstream areas (Stalnaker et al.,
2007; Schoenbaum et al., 2009; Takahashi et al., 2009), including the VS (Haber et al., 1995). Interestingly, animal studies found that intact OFC function is needed to learn from unexpected outcomes, i.e., from RPEs (Takahashi et al., 2009), and lesions in the OFC led to alterations of activity in dopaminergic neurons (Takahashi et al., 2011) and changes in striatal dopamine levels (Clarke et al., 2014), while stimulation of the VS influenced OFC activity and possibly connectivity (Ewing and Grace, 2013). Furthermore, it has been hypothesized that the OFC contributes information about current external states and is therefore crucially involved in the model-based aspect of learning (Takahashi et al., 2011). These findings suggest that the reduced OFC activation in participants with a high level of aberrant salience attribution might be related to the reduced RPE coding in the VS.

Limitations include that, because of our correlational approach, we cannot make any claims about causality. We focused here on the relationship between task-relevant, adaptive RPE signals during reinforcement learning and aberrant salience attribution measures. We did not measure brain activation related to aberrant salience attribution during the SAT. Roiser et al. (2010) found VS activation in response to adaptive salience during the SAT, i.e., the contrast of high-probability versus low-probability cues, in healthy participants, but no VS activation elicited by aberrant salience attribution to neutral cues. In another study, explicit aberrant salience ratings correlated positively with VS activation to irrelevant cue features across individuals (Roiser et al., 2013). Thus, the relation between task-relevant neural learning signals such as VS RPE (Roiser et al., 2013) and neural correlates of task-irrelevant, aberrant signals remains to be established. Studies in prodromal subjects found increased dopamine levels in the associative, dorsal striatum (Howes et al., 2009; Fusar-Poli et al., 2010). We did not obtain any differences in this striatal subdivision in this sample of healthy participants in relation to aberrant salience. However, an exploratory analysis revealed an association between aberrant salience attribution and presynaptic dopamine levels using values derived from the peak of RPE signal, which was located in the VS. This finding suggests an interdependence of dopamine levels and salience attribution given the assumption that RPEs and salience are associated concepts, but has to be replicated in an independent sample. Whether this result is related to the finding of increased dopamine levels in prodromal and schizophrenic subjects remains to be elucidated.

In conclusion, our findings are in line with predictions of the aberrant salience account of psychosis (Heinz, 2002; Kapur, 2003; Heinz and Schlagenhauf, 2010; Winton-Brown et al., 2014): we provide evidence that the attribution of salience to irrelevant cues is associated with reduced encoding of RPEs in the VS and OFC during reinforcement learning and with increased presynaptic dopamine levels in the VS region associated with RPE encoding. Here, obtained in healthy subjects, the same relationship may contribute to the development of positive symptoms in the psychotic disease spectrum.

\section{References}

Abi-Dargham A, Rodenhiser J, Printz D, Zea-Ponce Y, Gil R, Kegeles LS, Weiss R, Cooper TB, Mann JJ, Van Heertum RL, Gorman JM, Laruelle M (2000) Increased baseline occupancy of D2 receptors by dopamine in schizophrenia. Proc Natl Acad Sci USA 97:8104-8109. CrossRef Medline

Ashburner J, Friston KJ (2005) Unified segmentation. Neuroimage 26:839851. CrossRef Medline

Bay-Richter C, O’Tuathaigh CM, O’Sullivan G, Heery DM, Waddington JL, Moran PM (2009) Enhanced latent inhibition in dopamine receptor- 
deficient mice is sex-specific for the D1 but not D2 receptor subtype: implications for antipsychotic drug action. Int J Neuropsychopharmacol 12:403-414. Medline

Boureau YL, Dayan P (2011) Opponency revisited: competition and cooperation between dopamine and serotonin. Neuropsychopharmacology 36:74-97. CrossRef Medline

Clarke HF, Cardinal RN, Rygula R, Hong YT, Fryer TD, Sawiak SJ, Ferrari V, Cockcroft G, Aigbirhio FI, Robbins TW, Roberts AC (2014) Orbitofrontal dopamine depletion upregulates caudate dopamine and alters behavior via changes in reinforcement sensitivity. J Neurosci 34:7663-7676. CrossRef Medline

Corlett PR, Fletcher PC (2012) The neurobiology of schizotypy: frontostriatal prediction error signal correlates with delusion-like beliefs in healthy people. Neuropsychologia 50:3612-3620. CrossRef Medline

Corlett PR, Murray GK, Honey GD, Aitken MR, Shanks DR, Robbins TW, Bullmore ET, Dickinson A, Fletcher PC (2007) Disrupted predictionerror signal in psychosis: evidence for an associative account of delusions. Brain 130:2387-2400. CrossRef Medline

D'Ardenne K, McClure SM, Nystrom LE, Cohen JD (2008) BOLD responses reflecting dopaminergic signals in the human ventral tegmental area. Science 319:1264-1267. CrossRef Medline

Deserno L, Boehme R, Heinz A, Schlagenhauf F (2013) Reinforcement learning and dopamine in schizophrenia: dimensions of symptoms or specific features of a disease group? Front Psychiatry 4:172. CrossRef Medline

Deserno L, Huys QJ, Boehme R, Buchert R, Heinze HJ, Grace AA, Dolan RJ, Heinz A, Schlagenhauf F (2015) Ventral striatal dopamine reflects behavioral and neural signatures of model-based control during sequential decision making. Proc Natl Acad Sci U S A 112:1595-1600. CrossRef Medline

Egerton A, Chaddock CA, Winton-Brown TT, Bloomfield MA, Bhattacharyya S, Allen P, McGuire PK, Howes OD (2013) Presynaptic striatal dopamine dysfunction in people at ultra-high risk for psychosis: findings in a second cohort. Biol Psychiatry 74:106-112. CrossRef Medline

Ewing SG, Grace AA (2013) Long-term high frequency deep brain stimulation of the nucleus accumbens drives time-dependent changes in functional connectivity in the rodent limbic system. Brain Stimul 6:274-285. CrossRef Medline

First MB, Spitzer RL, Gibbon M, Williams J (2001) Structured clinical interview for DSM-IV-TR Axis I disorders, research version, patient edition with psychotic screen (SCID-I/P W/PSY SCREEN). New York: New York State Psychiatric Institute.

Fusar-Poli P, Howes OD, Allen P, Broome M, Valli I, Asselin MC, Grasby PM, McGuire PK (2010) Abnormal frontostriatal interactions in people with prodromal signs of psychosis: a multimodal imaging study. Arch Gen Psychiatry 67:683-691. CrossRef Medline

Goto Y, Grace AA (2008) Limbic and cortical information processing in the nucleus accumbens. Trends Neurosci 31:552-558. CrossRef Medline

Grace AA (2012) Dopamine system dysregulation by the hippocampus: implications for the pathophysiology and treatment of schizophrenia. Neuropharmacology 62:1342-1348. CrossRef Medline

Gradin VB, Kumar P, Waiter G, Ahearn T, Stickle C, Milders M, Reid I, Hall J, Steele JD (2011) Expected value and prediction error abnormalities in depression and schizophrenia. Brain 134:1751-1764. CrossRef Medline

Greenberg BD, Murphy DL, Rasmussen SA (2000) Neuroanatomically based approaches to obsessive-compulsive disorder. Neurosurgery and transcranial magnetic stimulation. Psychiatr Clin North Am 23:671-686, xii. CrossRef Medline

Haber SN, Kunishio K, Mizobuchi M, Lynd-Balta E (1995) The orbital and medial prefrontal circuit through the primate basal ganglia. J Neurosci 15:4851-4867. Medline

Heinz A (2002) Dopaminergic dysfunction in alcoholism and schizophrenia-psychopathological and behavioral correlates. Eur Psychiatry 17:9-16. CrossRef Medline

Heinz A, Schlagenhauf F (2010) Dopaminergic dysfunction in schizophrenia: salience attribution revisited. Schizophr Bull 36:472-485. CrossRef Medline

Howes OD, Kapur S (2009) The dopamine hypothesis of schizophrenia: version III-the final common pathway. Schizophr Bull 35:549-562. CrossRef Medline

Howes OD, Montgomery AJ, Asselin MC, Murray RM, Valli I, Tabraham P, Bramon-Bosch E, Valmaggia L, Johns L, Broome M, McGuire PK, Grasby
PM (2009) Elevated striatal dopamine function linked to prodromal signs of schizophrenia. Arch Gen Psychiatry 66:13-20. CrossRef Medline Howes OD, Kambeitz J, Kim E, Stahl D, Slifstein M, Abi-Dargham A, Kapur S (2012) The nature of dopamine dysfunction in schizophrenia and what this means for treatment. Arch Gen Psychiatry 69:776-786. CrossRef Medline

Huys QJ, Cools R, Gölzer M, Friedel E, Heinz A, Dolan RJ, Dayan P (2011) Disentangling the roles of approach, activation and valence in instrumental and pavlovian responding. PLoS Comput Biol 7:e1002028. CrossRef Medline

Huys QJ, Eshel N, O’Nions E, Sheridan L, Dayan P, Roiser JP (2012) Bonsa trees in your head: how the pavlovian system sculpts goal-directed choices by pruning decision trees. PLoS Comput Biol 8:e1002410. CrossRef Medline

Jackowski AP, Araújo Filho GM, Almeida AG, Araújo CM, Reis M, Nery F, Batista IR, Silva I, Lacerda AL (2012) The involvement of the orbitofrontal cortex in psychiatric disorders: an update of neuroimaging findings. Rev Bras Psiquiatr 34:207-212. CrossRef Medline

Kapur S (2003) Psychosis as a state of aberrant salience: a framework linking biology, phenomenology, and pharmacology in schizophrenia. Am J Psychiatry 160:13-23. CrossRef Medline

Kehagia AA, Murray GK, Robbins TW (2010) Learning and cognitive flexibility: frontostriatal function and monoaminergic modulation. Curr Opin Neurobiol 20:199-204. CrossRef Medline

Kringelbach ML, Rolls ET (2004) The functional neuroanatomy of the human orbitofrontal cortex: evidence from neuroimaging and neuropsychology. Prog Neurobiol 72:341-372. CrossRef Medline

Lodge DJ, Grace AA (2007) Aberrant hippocampal activity underlies the dopamine dysregulation in an animal model of schizophrenia. J Neurosci 27:11424-11430. CrossRef Medline

Malchow B, Hasan A, Meyer K, Schneider-Axmann T, Radenbach KE, Gruber O, Reith W, McIntosh AM, Schmitt A, Falkai P, Wobrock T (2015) Family load impacts orbitofrontal volume in first-episode schizophrenia. Psychiatry Res 232:130-133. CrossRef Medline

Martinez D, Slifstein M, Broft A, Mawlawi O, Hwang DR, Huang Y, Cooper T, Kegeles L, Zarahn E, Abi-Dargham A, Haber SN, Laruelle M (2003) Imaging human mesolimbic dopamine transmission with positron emission tomography. Part II: amphetamine-induced dopamine release in the functional subdivisions of the striatum. J Cereb Blood Flow Metab 23: 285-300. CrossRef Medline

Matsumoto M, Hikosaka O (2009) Two types of dopamine neuron distinctly convey positive and negative motivational signals. Nature 459: 837-841. CrossRef Medline

Matsumoto M, Takada M (2013) Distinct representations of cognitive and motivational signals in midbrain dopamine neurons. Neuron 79:10111024. CrossRef Medline

Meador-Woodruff JH, Haroutunian V, Powchik P, Davidson M, Davis KL, Watson SJ (1997) Dopamine receptor transcript expression in striatum and prefrontal and occipital cortex. Focal abnormalities in orbitofrontal cortex in schizophrenia. Arch Gen Psychiatry 54:1089-1095. CrossRef Medline

Menzies L, Chamberlain SR, Laird AR, Thelen SM, Sahakian BJ, Bullmore ET (2008) Integrating evidence from neuroimaging and neuropsychological studies of obsessive-compulsive disorder: the orbitofronto-striatal model revisited. Neurosci Biobehav Rev 32:525-549. CrossRef Medline

Mizrahi R, Addington J, Rusjan PM, Suridjan I, Ng A, Boileau I, Pruessner JC, Remington G, Houle S, Wilson AA (2012) Increased stress-induced dopamine release in psychosis. Biol Psychiatry 71:561-567. CrossRef Medline

Montague PR, Dayan P, Sejnowski TJ (1996) A framework for mesencephalic dopamine systems based on predictive Hebbian learning. J Neurosci 16:1936-1947. Medline

Murray GK, Corlett PR, Clark L, Pessiglione M, Blackwell AD, Honey G, Jones PB, Bullmore ET, Robbins TW, Fletcher PC (2008) Substantia nigra/ventral tegmental reward prediction error disruption in psychosis. Mol Psychiatry 13:239, 267-276. CrossRef Medline

Nagy H, Levy-Gigi E, Somlai Z, Takáts A, Bereczki D, Kéri S (2012) The effect of dopamine agonists on adaptive and aberrant salience in Parkinson's disease. Neuropsychopharmacology 37:950-958. CrossRef Medline

O'Callaghan MJ, Bay-Richter C, O’Tuathaigh CM, Heery DM, Waddington JL, Moran PM (2014) Potentiation of latent inhibition by haloperidol and clozapine is attenuated in Dopamine D2 receptor (Drd-2)-deficient 
mice: do antipsychotics influence learning to ignore irrelevant stimuli via both Drd-2 and non-Drd-2 mechanisms? J Psychopharmacol 28:973977. CrossRef Medline

O’Doherty JP, Dayan P, Friston K, Critchley H, Dolan RJ (2003) Temporal difference models and reward-related learning in the human brain. Neuron 38:329-337. CrossRef Medline

Padoa-Schioppa C, Assad JA (2006) Neurons in the orbitofrontal cortex encode economic value. Nature 441:223-226. CrossRef Medline

Patlak CS, Blasberg RG (1985) Graphical evaluation of blood-to-brain transfer constants from multiple-time uptake data. Generalizations. J Cereb Blood Flow Metab 5:584-590. CrossRef Medline

Peters ER, Joseph SA, Garety PA (1999) Measurement of delusional ideation in the normal population: introducing the PDI (Peters et al. Delusions Inventory). Schizophr Bull 25:553-576. CrossRef Medline

Poletti M, Frosini D, Pagni C, Baldacci F, Lucetti C, Del Dotto P, Ceravolo R, Bonuccelli U (2014) A pilot psychometric study of aberrant salience state in patients with Parkinson's disease and its association with dopamine replacement therapy. Neurol Sci 35:1603-1605. CrossRef Medline

Raine A (1991) The SPQ: a scale for the assessment of schizotypal personality based on DSM-III-R criteria. Schizophr Bull 17:555-564. CrossRef Medline

Robinson TE, Berridge KC (1993) The neural basis of drug craving: an incentive-sensitization theory of addiction. Brain Res Brain Res Rev 18: 247-291. CrossRef Medline

Roesch MR, Olson CR (2004) Neuronal activity related to reward value and motivation in primate frontal cortex. Science 304:307-310. CrossRef Medline

Roiser JP, Stephan KE, den Ouden HE, Barnes TR, Friston KJ, Joyce EM (2009) Do patients with schizophrenia exhibit aberrant salience? Psychol Med 39:199-209. CrossRef Medline

Roiser JP, Stephan KE, den Ouden HE, Friston KJ, Joyce EM (2010) Adaptive and aberrant reward prediction signals in the human brain. Neuroimage 50:657-664. CrossRef Medline

Roiser JP, Howes OD, Chaddock CA, Joyce EM, McGuire P (2013) Neural and behavioral correlates of aberrant salience in individuals at risk for psychosis. Schizophr Bull 39:1328-1336. CrossRef Medline

Romaniuk L, Honey GD, King JR, Whalley HC, McIntosh AM, Levita L, Hughes M, Johnstone EC, Day M, Lawrie SM, Hall J (2010) Midbrain activation during Pavlovian conditioning and delusional symptoms in schizophrenia. Arch Gen Psychiatry 67:1246-1254. CrossRef Medline
Schlagenhauf F, Sterzer P, Schmack K, Ballmaier M, Rapp M, Wrase J, Juckel G, Gallinat J, Heinz A (2009) Reward feedback alterations in unmedicated schizophrenia patients: relevance for delusions. Biol Psychiatry 65: 1032-1039. CrossRef Medline

Schlagenhauf F, Huys QJ, Deserno L, Rapp MA, Beck A, Heinze HJ, Dolan R, Heinz A (2014) Striatal dysfunction during reversal learning in unmedicated schizophrenia patients. Neuroimage 89:171-180. CrossRef Medline

Schmidt K, Roiser JP (2009) Assessing the construct validity of aberrant salience. Front Behav Neurosci 3:58. CrossRef Medline

Schoenbaum G, Roesch MR, Stalnaker TA, Takahashi YK (2009) A new perspective on the role of the orbitofrontal cortex in adaptive behaviour. Nat Rev Neurosci 10:885-892. CrossRef Medline

Schönberg T, Daw ND, Joel D, O’Doherty JP (2007) Reinforcement learning signals in the human striatum distinguish learners from nonlearners during reward-based decision making. J Neurosci 27:12860-12867. CrossRef Medline

Schultz W, Dayan P, Montague PR (1997) A neural substrate of prediction and reward. Science 275:1593-1599. CrossRef Medline

Stalnaker TA, Franz TM, Singh T, Schoenbaum G (2007) Basolateral amygdala lesions abolish orbitofrontal-dependent reversal impairments. Neuron 54:51-58. CrossRef Medline

Sutton R, Barto AG (1998) Reinforcement learning: an introduction. Cambridge, MA: MIT.

Takahashi YK, Roesch MR, Stalnaker TA, Haney RZ, Calu DJ, Taylor AR, Burke KA, Schoenbaum G (2009) The orbitofrontal cortex and ventral tegmental area are necessary for learning from unexpected outcomes. Neuron 62:269-280. CrossRef Medline

Takahashi YK, Roesch MR, Wilson RC, Toreson K, O’Donnell P, Niv Y, Schoenbaum G (2011) Expectancy-related changes in firing of dopamine neurons depend on orbitofrontal cortex. Nat Neurosci 14:15901597. CrossRef Medline

Ullsperger M, Danielmeier C, Jocham G (2014) Neurophysiology of performance monitoring and adaptive behavior. Physiol Rev 94:35-79. CrossRef Medline

Weiner I, Arad M (2009) Using the pharmacology of latent inhibition to model domains of pathology in schizophrenia and their treatment. Behav Brain Res 204:369-386. CrossRef Medline

Winton-Brown TT, Fusar-Poli P, Ungless MA, Howes OD (2014) Dopaminergic basis of salience dysregulation in psychosis. Trends Neurosci 37: 85-94. CrossRef Medline 